\title{
Article \\ Sliding Group Window with Rebacking off for Collision Avoidance in High-Efficiency Wireless Networks
}

\author{
Alaa Omran Almagrabi ${ }^{1,+}{ }^{\text {, }}$ ashid Ali ${ }^{2,+} \mathbb{D}$, Yasser Difulah Al-Otaibi ${ }^{3} \mathbb{D}$, Hadi Mohsen Oqaibi ${ }^{1}$ \\ and Tahir Khurshaid $4, *$ (D)
}

1 Department of Information Systems, Faculty of Computing and Information Technology, King Abdulaziz University, Jeddah 21589, Saudi Arabia; aalmagrabi3@kau.edu.sa (A.O.A.); hoqaibi@kau.edu.sa (H.M.O.)

2 School of Intelligent Mechatronics Engineering, Sejong University, Seoul 05006, Korea; rashidali@sejong.ac.kr

3 Department of Information Systems, Faculty of Computing and Information Technology in Rabigh,

King Abdulaziz University, Jeddah 21589, Saudi Arabia; yalotaibi@kau.edu.sa

4 Department of Electrical Engineering, Yeungnam University, Gyeongsan 38541, Korea

* Correspondence: tahir@ynu.ac.kr; Tel.: +82-2-6935-2608

+ A.O.A. and R.A. have equally contributed as First Author.

Citation: Almagrabi, A.O.; Ali, R.; Al-Otaibii, Y.D.; Oqaibi, H.M.;

Khurshaid, T. Sliding Group Window with Rebacking off for Collision Avoidance in High-Efficiency Wireless Networks. Mathematics 2021, 9, 2461. https://doi.org/10.3390/ math9192461

Academic Editor: Daniel-Ioan Curiac

Received: 29 August 2021

Accepted: 26 September 2021

Published: 2 October 2021

Publisher's Note: MDPI stays neutral with regard to jurisdictional claims in published maps and institutional affiliations.

Copyright: (c) 2021 by the authors. Licensee MDPI, Basel, Switzerland. This article is an open access article distributed under the terms and conditions of the Creative Commons Attribution (CC BY) license (https:/ / creativecommons.org/licenses/by/ $4.0 /)$.

\begin{abstract}
It is difficult for wireless local area networks (WLANs), IEEE 802.11ax high-efficiency WLAN (HEW), to join next-generation innovations such as 5th generation (5G) and Internet of Things (IoT) because they still have their conventional channel access mechanism as their essential medium access control (MAC) protocol. The MAC protocol uses a traditional binary exponential backoff (BEB) algorithm to access channel resources that depend on the noncognitive increment of contention parameters for collision avoidance. In $\mathrm{BEB}$, the collision issue increases with the increase in connected devices in the network due to a fixed contention window size. The larger the size of the network, the larger the collision in the network. To avoid such a circumstance, in this paper, we propose a sliding group window (sGW) mechanism dependent on collision-point assessment in order to improve the performance of MAC protocol for HEW. The proposed algorithm additionally presents a rebacking off for collision avoidance (ReBOCA) system for sGW, which combines the uniform dispersion of the contention parameters. This variation of an ordinary backoff algorithm permits the reasonable sliding of the user groups in the case of collision. The algorithm explicitly accounts for the peculiarities of dense environments and backward compatibility. Key aspects of the proposed solution include collision-point estimation, rebacking off for collision distribution convergence for fair treatment, and adaptive sliding of group windows to mitigate contention unfairness. We further formulated a closed-form Markov chain model for the performance analysis of our proposed sGW with ReBOCA scheme. Theoretical and practical results prove that our proposed scheme achieved maximal efficiency, even under dense environments. An increase in throughput with a lower packet collision probability was achieved with the proposed mechanism, and the efficiency increased as the number of contending stations increased than compared to traditional BEB performance. Our proposed ReBOCA mechanism enhanced network throughput by $38.18 \%$ than compared to the conventional BEB mechanism.
\end{abstract}

Keywords: applied mathematics; random access; channel access; wireless local area network; resource allocation; wifi

\section{Introduction}

Wireless local area networks (WLAN), standard name IEEE 802.11, require cuttingedge wireless advancements such as fifth-generation (5G) and beyond (B5G) networks. The development of the 5G/B5G period has guaranteed endless time availability and artificial-intelligence (AI)-enabled automation. The 5G/B5G technologies aim to support several fold gains in capacity, associations for at least a hundred billion connections, and tens of gigabits per second of individual user quality of experience (QoE) capable of 
ultrareliable low-latency communication (URLLC) [1]. To help in the enormous URLLC requirements, the IEEE 802.11ax high-efficiency WLAN (HEW) is promising advancing 5G/B5G radio access networks (RANs) to the unlicensed band [2].

One of the most critical issues for 5G/B5G is to meet URLLC requirements for realtime applications. There exist several potential techniques in the physical (PHY) layer of a WLAN to achieve this ambiguous goal for 5G/B5G systems. For example, powerful error correction based on polar codes in 5G new radio (5G NR) [3] and low-density parity check (LDPC) codes [4] may help in enhancing promising PHY layer transmission solutions. Moreover, the mathematical analysis of mobile transmission channels raises more challenges due to highly dynamic network situations. A hybrid decoded amplify-forward (HDAF) relay mechanism with transmission antenna selection may be favorable [5]. However, a HEW station (STA) faces massive collisions to reach unlicensed wireless networks, especially for a high number of associated STAs. The WLAN medium access control (MAC) layer is fundamentally based on the amplification of the channel resources by using a carrier sense multiple access with collision avoidance (CSMA/CA) mechanism for STAs to channel access [6] in order to achieve the best wireless medium used through reasonable fair access in the WLANs, with the reliably growing density of STAs. The currently used CSMA/CA mechanism plays a crucial part for the upcoming 5G/B5G [7]. The binary exponential backoff (BEB) algorithm is the core of the CSMA/CA mechanism [8]. The BEB generates a uniform random backoff number for channel contention in a WLAN. First, a STA chooses a uniform random value $b$ from a predefined minimal contention window $\left[0, C W_{\text {min }}\right]$. After each collision, the current $C W$ exponentially increases until it reaches the maximal allowable $C W$ size (after $m$ number of collisions), which is $C W_{\max }=2^{m} \times C W_{\min }$, for $m$ greatest number of backoff stages $(i)$, which is $i \in(0, m)$. When a STA effectively communicates its information packet, the current $C W$ size, which is $C W_{i}$, is reset to the initial $\mathrm{CW}_{\text {min }}$.

\subsection{Problem Statement}

For a WLAN with a substantial network load, resetting the value of the backoff to zero and $C W$ to its initial value $C W_{\min }$ after successful packet transmission brings more collisions and network performance degradation because of an increment in probability to choose comparable backoff value $b$ for a given number of devices (possibly large due to denser deployments) [9]. Similarly, for fewer competing STAs, the blind increment of $\mathrm{CW}_{i}$ for collision avoidance creates a longer delay because of the larger range for choosing $b$. This blind increment or decrease in the backoff window is more wasteful in the exceptionally dense WLANs proposed for HEW on the grounds that the probability of collision increments with the increasing number of STAs. Hence, the current channel access scheme (that is, CSMA/CA) does not permit WLANs to accomplish high throughput in exceptionally dense conditions. There are several related research works in the literature $[6,10-13]$ that propose to handle these issues (a detailed discussion can be found further below). However, a concrete solution for collision avoidance due to the increase in the number of connected STAs is yet to be explored. Thus, to withstand this issue, WLANs need a more efficient backoff scheme to guarantee improved user QoE.

\subsection{Motivation for the Proposed Solution}

In this paper, we propose the replacement of the standard backoff algorithm BEB with a sliding group window (sGW) algorithm, which depends on collision-point assessment to improve the effectiveness of MAC layer resource allocation for HEW. The proposed mechanism additionally presents a rebacking off for the collision-avoidance (ReBOCA) algorithm for $\mathrm{sGW}$, which converges the uniform random distribution of $\mathrm{CW}$. The proposed ReBOCA system, dependent on sGW, permits a reasonable sliding of the contending groups if there is a collision in the network. The proposed algorithm unequivocally represents the characteristics of dense network conditions and backward compatibility. Key aspects of the proposed solution include collision-point estimation, rebacking off for collision 
distribution convergence for fair treatment, and adaptive sliding of group windows to mitigate contention unfairness. We further formulate a closed form two-dimensional Markov Chain (TDMC) model for the performance analysis of our proposed sGW with ReBOCA scheme.

The paper structure is as follows. In Section 2, related research works are presented, highlighting similar work. Section 3 describes the proposed ReBOCA algorithm. Section 4 presents an analytical modeling of IEEE 802.11 DCF for our proposed ReBOCA algorithm. The proposed model is validated in Section 5. Section 6 concludes our findings and results and presents future work considerations.

\section{Related Research Work}

Many studies have investigated methods to adaptively and efficiently increase or decrease the backoff contention window. In $[10,11]$, throughput was expanded in saturated and unsaturated data traffic conditions by keeping the CW from resetting to its base threshold after each successful transmission. In their proposed enhanced collision avoidance (ECA) mechanism, a deterministic backoff value $B=\frac{C W_{\min }}{2}$ could be utilized instead of resetting $C W_{c u r}$ to $C W_{\text {min }}$, which reduces the chances of collisions for STAs that were successful in their earlier transmission. ECA guarantees that more channel time is spent on successful transmission instead of recovering due to network collisions, which expands the throughput of the WLAN [11]. However, this improvement results in the impairment of diminished short-term fairness, since STAs that experience several collisions are constrained to stay at a higher backoff stage without knowing the density of the network and are contrarily rewarded by less regular transmissions. Moreover, ECA is efficient for smaller networks until the number of STAs is less than the deterministic cycle length $\frac{C W_{\min }}{2}$. Similarly, Ye et al. [12] presented an exponential increase-exponential decrease (EIED) backoff algorithm where the value of $C W_{c u r}$ exponentially increased for each collision and reduces to the halved value after each successful packet transmission. It is not enough to control the increase or decrease in $C W_{c u r}$ because there must be an adaptive and optimized mechanism to adjust the backoff contention window. A more adaptive method to adjust backoff $C W$ is to obtain the practical collision probability that an STA faces during transmission attempts. This critical issue of the critical channel collision problem due to the massive number of contending STAs to access a single channel for transmission was solved with the help of a practical channel-observation-based scaled backoff (COSB) mechanism in [6,13]. The COSB [6] algorithm ensures high-throughput and low channel access delay by decreasing the number of impacts in the direct access mechanism in both saturated and unsaturated network conditions.

\section{Rebacking-off-Based Collision-Avoidance Algorithm}

In this part, we clarify our proposed ReBOCA mechanism. The proposed ReBOCA mechanism presents the defense for the suitability of CSMA/RCA as a future substitution to the traditional CSMA/CA. As mentioned in Section 2, a few works proposed changes to CSMA/CA regardless of throughput improvement, but none of the proposed adjustments have yet to embraced by the norm. To replace traditional CSMA/CA, a candidate mechanism both needs to provide performance advantages as far as throughput and fairness and should be backward-compatible with the current CSMA/CA. It needs to support a large number of contending STAs, and it needs to be a simple evolution in terms of implementation so that it can easily be replaced and reduce the time to market. The CSMA/RCA mechanism with the ReBOCA algorithm satisfies the four above requirements, which makes it a suitable candidate to replace CSMA/CA in the upcoming revisions of the standard. Moreover, as the CSMA/RCA follows a group-based backing-off mechanism, it is also compatible with the QoS-based WLAN standards where traffic is prioritized according to groups. 


\section{CSMA/RCS with Re-BOCA}

Algorithm 1 depicts the ordinary CSMA/CA mechanism that is currently utilized in WLAN networks for channel access. At the point when a STA joins the channel contention, it initiates retry counter $r=0$ and backoff stage $s=0$. Randomly selected backoff counter $b$ is from the initial contention window $C W_{\text {min }}$. The retry counter and backoff stage counter increment after each collision. As an outcome of the increased backoff stage, a double $C W$ is utilized. There is a maximal backoff stage $S$, and most extreme retry limits $R$ are indicated by the standard. At the point when the number of transmissions reaches the upper retry limit, the data frame is disposed of. $r$ and $s$ are reset to the initial values, and $b$ is regenerated. The pseudocode performs the backoff decrements action in lines 6-9.

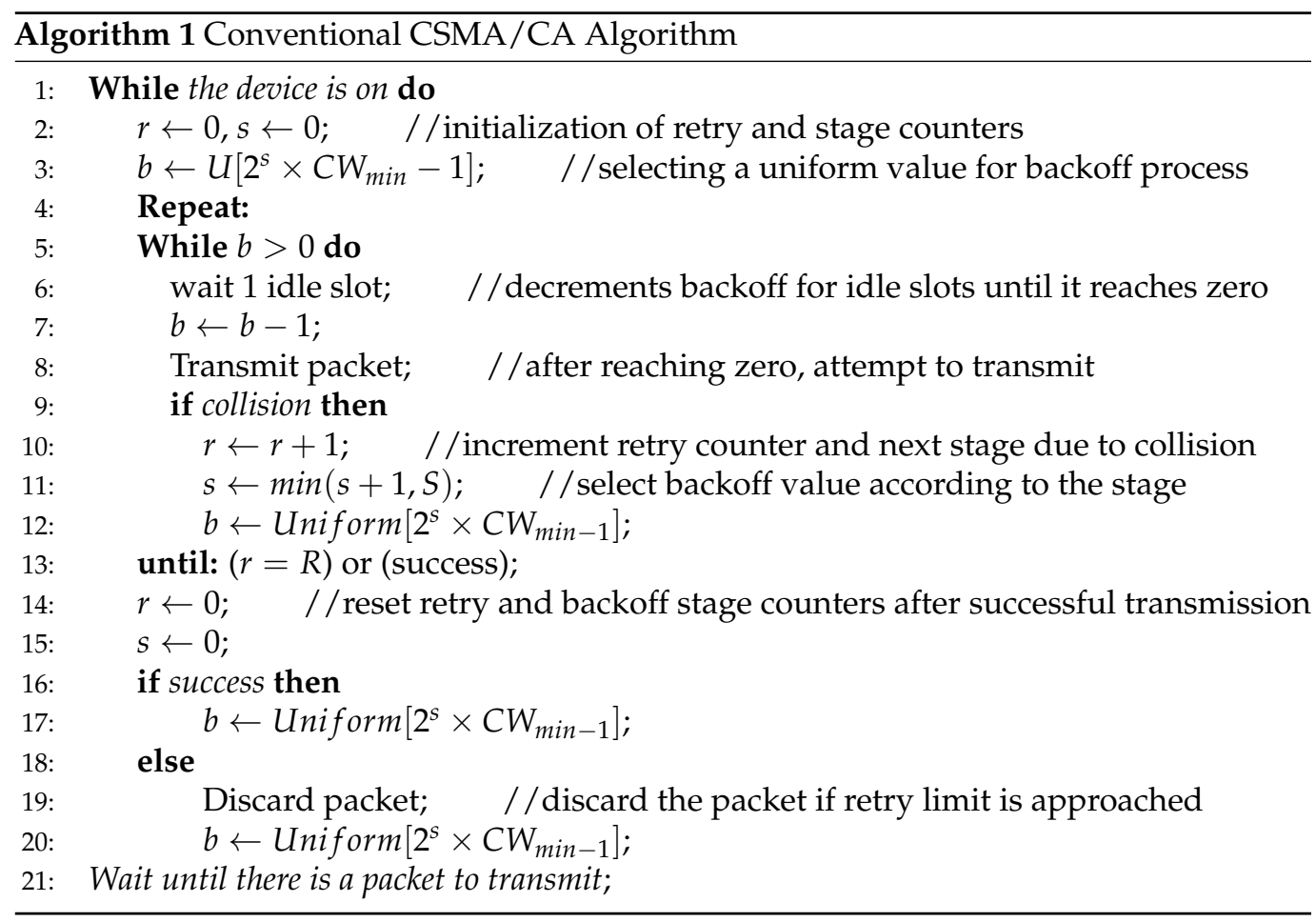

There is one change in the CSMA/RCA compared to the legacy CSMA/CA protocol. The backoff values of $C W$ are divided into four groups: $A, B, C$, and $D$. This simple modification converts CSMA/CA into CSMA/RCA. If the initial $C W_{\text {min }}$ size is 32 , then the groups are formed as $A=\{0-7\}, B=\{8-15\}, C=\{16-23\}$, and $D=\{24-31\}$. At the point when a STA joins the contention, it introduces the retry counter as $r=0$ and the backoff stage as $s=0$. Backoff counter $b$ is instated by utilizing a uniform random value and the minimal CW CW $\mathrm{CW}_{\text {min }}$ (for example, for our situation, 32). Every collision increments retry counter $r$ and backoff stage counter $s$. As an outcome of the incremented backoff stage, a larger $C W$ is utilized with equation $C W_{i}=2^{i} \times C W_{\text {min }}$ for each $i$ th stage.

Algorithm 2 depicts the CSMA/RCA technique in which rebacking off is utilized at whatever point a STA contends for the channel. The changes for CSMA/CA are shown in lines 4-11 and 17-22, where a uniform random value of $b$ in CSMA/CA is replaced by a group-based CW in CSMA/RCA. At this point, the value of $s$ is zero, and the group size can be determined as follows:

$$
\operatorname{Size}\left[G^{g}\right]=2^{i} \times \frac{C W_{\min }}{4},
$$

where $g=\{A, B, C, D\}$, and they have values as $\{1,2,3,4\}$, and $i$ is the current backoff stage $i=\{0,1,2, \ldots, S\}$. Since $C W$ starts from 0 (e.g., if $C W=32$, its backoff selection 
range is (0-31), therefore, the lower bound of first group $A$ can be written as $G_{l}^{1}=0$; thus, to calculate the upper bounds of each group, we use following equation:

$$
G_{u}^{g}=g \times \operatorname{Size}\left[G^{g}\right]-1,
$$

where $g=\{1,2,3,4\}$ is the group number, and $u$ represents the upper bound. Once the upper bound of 1st group is calculated, the lower bounds of rest of the groups can easily be determined as follows:

$$
G_{l}^{g}=G_{u}^{(g-1)}+1,
$$

where $g=\{2,3,4\}$. For example, if $C W_{\min }=32$, then the group formation for the initial stage (i.e., $i=0$ ) can be written as Size $\left[G^{g}\right]=2^{0} \times \frac{32}{4}=8$; thus, the size of each group is 8 . We find the lower and upper bounds of each group as $G_{l}^{1}=0, G_{u}^{1}=1 \times 8-1=7$; thus, Group $A=\{0-7\}$. Similarly, $G_{l}^{2}=G_{u}^{1}+1=7+1=8$, and $G_{u}^{2}=2 \times 8-1=15$; thus, Group $B=\{8-15\}$. For Group $C, G_{l}^{3}=G_{u}^{2}+1=15+1=16$ and $G_{u}^{3}=3 \times 8-1=23$; thus, Group $C=\{16-23\}$. Lastly, for Group $D, G_{l}^{4}=G_{u}^{3}+1=23+1=24$ and $G_{u}^{4}=4 \times 8-1=31$; thus, Group $C=\{24-31\}$.

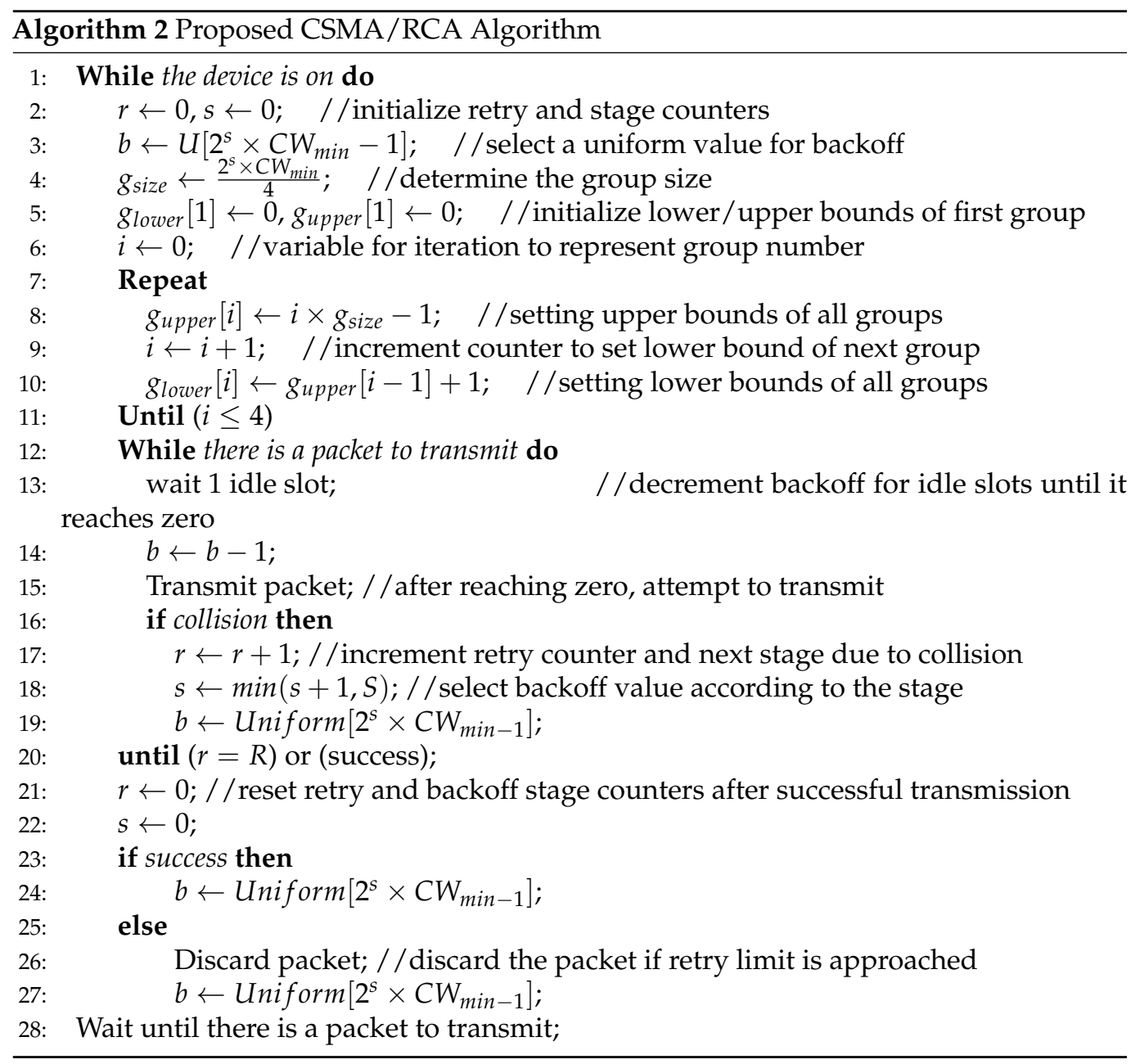

This particular choice of grouping improves fairness between new and legacy STAs, and even QoS-based STAs can be part of this contention as the groups are equivalent to the priority grouping in QoS-based schemes.

An STA joins the contention group according to the selected backoff uniform value and starts decrementing counter $b$ for idle slots. In a conventional CSMA/CA, STA starts transmitting if it successfully completes the backoff counter (that is, it reaches zero). In our modified ReBOCA process, the STA rebackoff reaches the lower bound of the group (that 
is, it selects the backoff value again from one group prior to the current) until it successfully finishes the backoff counter selected in last group $A$. For example, if a contending STA selects $b=19$, it joins group $C$, as shown in Figure 1. After selecting the group, STA starts decrementing the backoff counter until it reaches the edge of group $C$, that is, 16 . After successfully completing group $C$, it rebacks off and selects another backoff counter $r b_{B}$ from group $B=\{18-15\}$. Let us assume that STA picks a counter value at $r b_{B}=12$; it keeps sensing the channel and decrements the counter. After successfully completing this group, it performs rebackoff $r b_{A}$ in a similar manner in group $A=\{0-7\}$. Lastly, if the STA successfully completes group $A$, it starts transmitting. It resets the backoff stage after a successful data frame transmission.

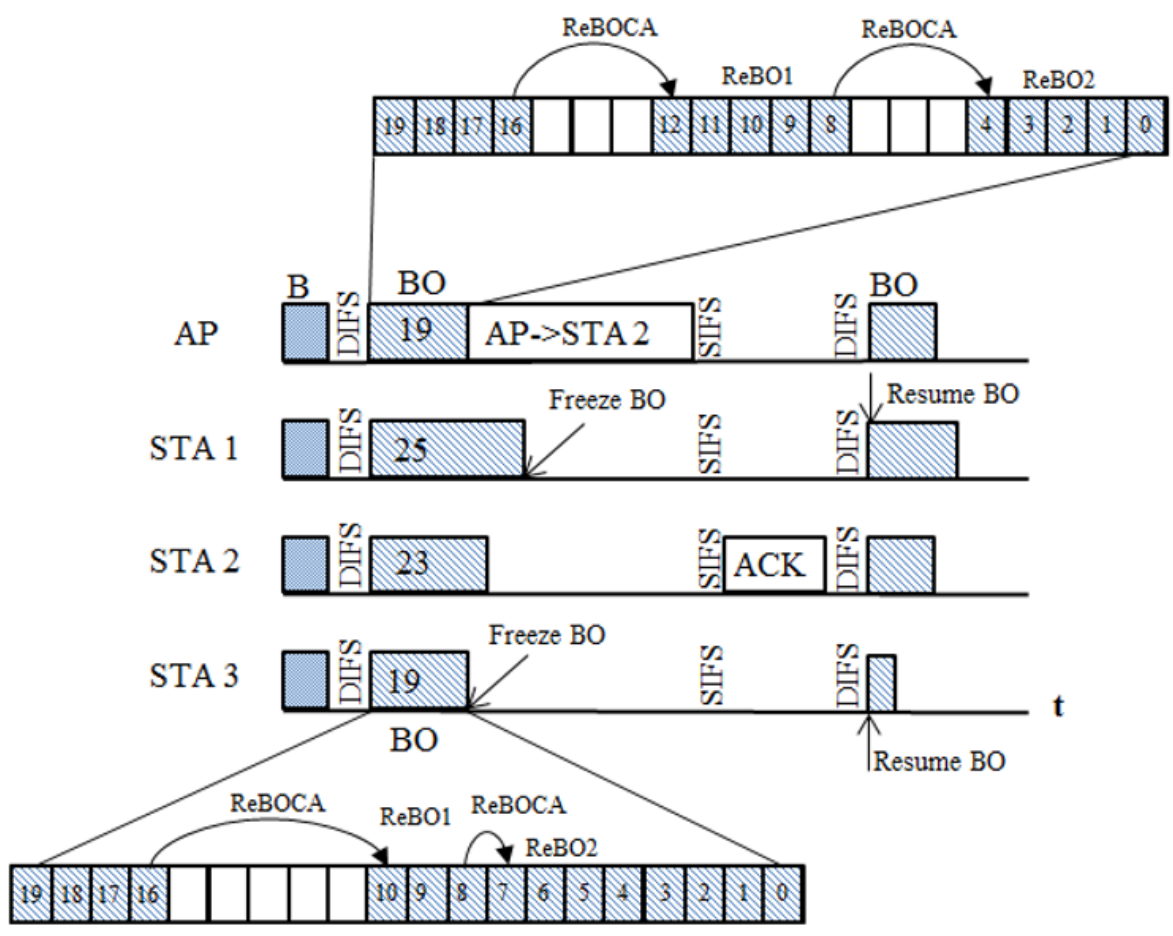

Figure 1. IEEE 802.11 DCF transmission procedure in CSMA/RCA with ReBOCA.

\section{Analytical Modeling of IEEE 802.11 DCF for ReBOCA}

We formulated the analytical modeling for the proposed ReBOCA mechanism with saturated throughput, with the assumption of ideal channel conditions, that is, no hidden STA and channel capture effects. In addition, the analytical model assumes the fixed number of connected STAs in the network. At first, we study the behavior of a specific STA in the WLAN with a discrete-time Markov chain model (DTMC) [14,15], and we obtain stationary probability of transmission $\tau$ for the selected STA. Since our proposed ReBOCA algorithm does not reset the backoff stage to zero after successful transmission, the data frame transmission attempt stays recursive inside the backoff stages.

In ReBOCA, the MAC protocol finishes its collision resolution process by using a sGW mechanism (theReBOCA BEB procedure) with probability $(1-p)$ and goes for a successful transmission of the given frame. In Figure 2, $m$ is used for the maximal number of retransmission stages due to collision, $W_{j}^{i}$ is for maximal CW size for the $i$ th stage due to collision in $j$ th group of $(i-1)^{\text {th }}$ stage, and $p$ is for the probability of the transmitted frame collision.

\subsection{Steady-State Probabilities of sGW-DTMC for ReBOCA}

Let $b_{i, j, k}$ be the steady-state probability of active state $(i, j, k)$ where $i$ is the number of retransmission limits and $i \in\{0,1,2, \ldots, m\}$, and $j$ is the group number from the previous collided stage and $j \in\left\{0,1,2, \ldots, g^{i-1}\right\}$, where $g^{i}$ determines the number of groups in 
stage. Lastly, the $k$ is the backoff value selected at the $i$ th stage, and $k \in\left(0, W_{j}^{i}-1\right), W_{j}^{i}$ is the maximal CW size in $i$ th stage, and it depends upon the collision from $j$ th group in $(i-1)^{t h}$ stage.

From Figure 2, we write the possible transactions as follows.

$$
\left\{\begin{array}{l}
P\{(0, j, k) \mid(i, 0,0)\}=\frac{1-p}{W_{g^{0}}^{0}}, \quad i \in(0, m), k \in\left(0, W_{g^{0}}^{0}-1\right), j \in\left\{0,1,2, \ldots, g^{0-1}\right\} \\
P\{(i, j, k) \mid(i-1,0,0)\}=\frac{p}{W_{j}^{i}}, \quad i \in(0, m), k \in\left(0, W_{j}^{i}-1\right), j \in\left\{0,1,2, \ldots, g^{i}-1\right\} \\
P\{(m, j, k) \mid(m-1,0,0)\}=\frac{p}{W_{j}^{m}}, \quad i \in(0, m), k \in\left(0, W_{j}^{m}-1\right), j \in\left\{0,1,2, \ldots, g^{i}-1\right\}
\end{array}\right.
$$

In the above set of equations, the first equation represents the manner in which, after a successful packet transmission begins with backoff stage 0 , the backoff is at first consistently selected in the range $\left(0, W_{g^{0}}^{0}-1\right)$, where $W_{g^{0}}^{0}-1$ is the $C W_{m i n}$, and $g^{0}$ is the initial number of groups for the first backoff stage. The other two conditions model the framework after a collision. In particular, as in the second condition of (4), when an unsuccessful transmission happens at backoff stage $i-1$, the backoff stage increments and the new backoff value are consistently selected in the range of $\left(0, W_{j}^{i}-1\right)$, where $W_{j}^{i}-1$ is the maximal CW size in $i$ th stage and it depends upon the collision from $j$ th group in $(i-1)^{\text {th }}$ stage. Lastly, the third case models that, once the backoff stage reaches value $m$, it is not increased, and the packet is simply discarded in the case of further unsuccessful transmission. At the beginning of each idle slot time, the backoff time is decremented, and we assume the following, as shown in Figure 3.

$$
P\{(0, j, k) \mid(0, j, k+1)\}=1, \quad i \in(0, m), k \in\left(0, W_{j}^{i}-1\right), j \in\left\{0,1,2, \ldots, g^{i}-1\right\}
$$

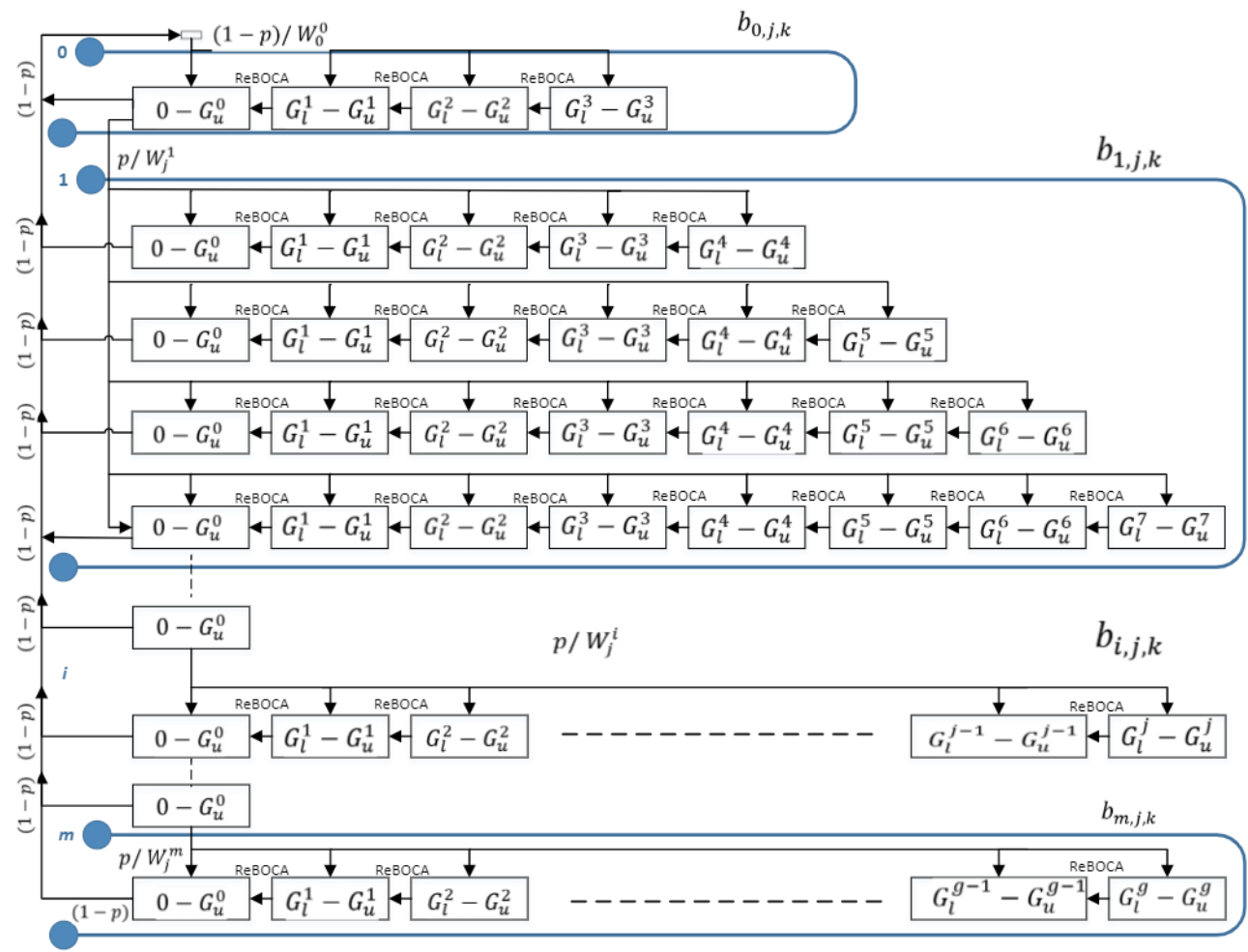

Figure 2. Discrete time Markov chain transition diagram of ReBOCA with sGW Model. 


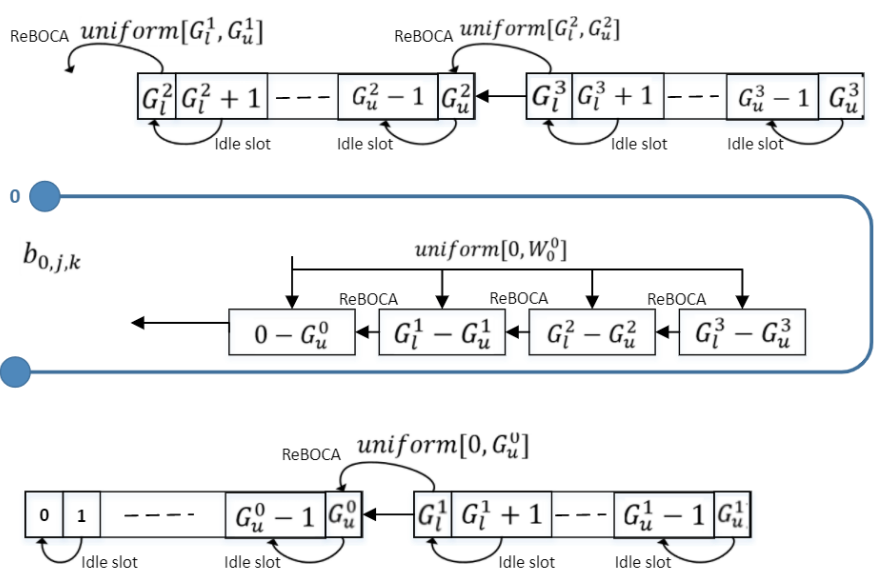

Figure 3. Transition diagram of backoff time decrements in ReBOCA.

As discussed earlier, the DTMC for sGW in Figure 2 is a two-dimensional DTMC; thus, for the equilibrium equations, we break the sGW-DTMC into multiple dimensions. Therefore, the equilibrium equations for such a multidimensional DTMC can be obtained as follows.

$$
\begin{aligned}
(1-p+p) \times b_{i, 0,0} & =p \times b_{i-1,0,0} \\
b_{i, 0,0} & =p \times b_{i-1,0,0}
\end{aligned}
$$

Similarly, we have the following.

$$
b_{i-1,0,0}=p \times b_{i-2,0,0} \rightarrow b_{1,0,0}=p \times b_{0,0,0}
$$

Hence, we can write the following.

$$
b_{i, 0,0}=p^{i} \times b_{0,0,0,} \quad 0<i<m \text {. }
$$

Since a packet is discarded after collision at Stage $b_{m, 0,0}$, we consider steady states for $b_{m, 0,0}$ as follows.

$$
\begin{array}{r}
(1-p) \times b_{m, 0,0}=p \times b_{m-1,0,0} \\
b_{m, 0,0}=\frac{p}{1-p} \times b_{m-1,0,0}
\end{array}
$$

Hence, we can write transition probability for the $m^{\text {th }}$ stage as follows.

$$
b_{m, 0,0}=\frac{p^{m}}{1-p} \times b_{0,0,0}
$$

Now, owing to the Markov chain regularities, for each $k \in\left(1, W_{j}^{i}-1\right)$, the stationary distribution for $\{s(t), g(t), b(t)\}$ can be written as follows:

$$
b_{i, j, k}=\frac{W_{j}^{i}-\sum_{j=0}^{g^{i}-1} \frac{G_{u}^{j}-k_{r}^{j}}{g_{s i z e}}}{W_{j}^{i}}\left\{\begin{array}{l}
(1-p) \times \sum_{l=0}^{m} b_{l, 0,0}, \quad i=0 \\
p \times b_{i-1,0,0}, \quad 0<i \leq m
\end{array} .\right.
$$


where $g_{\text {size, }} G_{u}^{j}$, and $k_{r}^{j}$ are the sizes of the groups, the upper bound of the $j$ th group, and the rebackoff value in the $j$ th group, respectively. Suppose $\sum_{j=0}^{g^{i}-1} \frac{G_{u}^{j}-k_{r}^{j}}{g_{s i z e}}=k^{\prime}$; thus, (8) can be written as follows.

$$
b_{i, j, k}=\frac{W_{j}^{i}-k^{\prime}}{W_{j}^{i}}\left\{\begin{array}{l}
(1-p) \times \sum_{l=0}^{m} b_{l, 0,0}, \quad i=0 \\
p \times b_{i-1,0,0,} \quad 0<i \leq m
\end{array} .\right.
$$

Thus, by Relations (6)-(9), all values $b_{i, j, k}$ are presented as functions of value $b_{0,0,0}$ and of collision probability $p$, where $b_{0,0,0}$ is lastly determined by using the normalization condition as follows.

$$
\begin{gathered}
1=\sum_{i=0}^{m} \sum_{k^{\prime}=0}^{W_{j}^{i}-1} b_{i, j, k^{\prime}}=\sum_{i=0}^{m} b_{i, 0,0} \sum_{k^{\prime}=0}^{W_{j}^{i}-1} \frac{W_{j}^{i}-k^{\prime}}{W_{j}^{i}}=\sum_{i=0}^{m} b_{i, 0,0} \frac{W_{j}^{i}+1}{2} \\
=\frac{1}{2}\left[\sum_{i=0}^{m-1} b_{i, 0,0} \times W_{j}^{i}+b_{m, 0,0} \times W_{j}^{m}+\frac{b_{0,0,0}}{1-p}\right]
\end{gathered}
$$

From the fact that $\sum_{i=0}^{m} b_{i, 0,0}=\frac{1}{1-p} \times b_{0,0,0}$ and we also know that $W_{g^{0}}^{0}=C W_{\text {min }}=W$ and $W_{j}^{i}=\left(2^{i} \times g^{0}-j\right) \times g_{s i z e}$, for all $i \in(1, m)$ where $g_{0}$ and $g_{\text {size }}$ are the number of groups in backoff stage 0 and size of each group, respectively. Thus, the following is the case.

$$
1=\frac{1}{2}\left[\sum_{i=0}^{m-1} b_{i, 0,0} \times\left(2^{i} \times g^{0}-j\right) \times g_{\text {size }}+\frac{p^{m}}{1-p} \times b_{0,0,0} \times\left(2^{m} \times g^{0}-j\right) \times g_{\text {size }}+\frac{b_{0,0,0}}{1-p}\right]
$$

$j$ is the group number of the previous stage where collision was happened; let $E[j]$ be the expected group number in $(i-1)^{t h}$ stage, and it can be written as $E[j]=\frac{2^{i-1} \times g^{0}}{2}$, hence, solving (11). In $b_{0,0,0}$, we obtain the following.

$$
1=\frac{b_{0,0,0}}{2}\left[\frac{3 \times W}{4}\left(\frac{1-(2 p)^{m}}{(1-2 p)}+\frac{(2 p)^{m}}{1-p}\right)+\frac{1}{1-p}\right]
$$

After several steps, we can write this as the following.

$$
b_{0,0,0}=\frac{8 \times(1-2 p) \times(1-p)}{(3 W+4) \times(1-2 p)+3 p \times W \times\left(1-(2 p)^{m}\right)}
$$

Since the probability of transmitting a frame depends on the value of collision probability $p$, let $\tau$ be the probability that a STA transmits in a randomly chosen slot time; then, regardless of the backoff stage, $\tau$ can be written as follows.

$$
\tau=\sum_{i=0}^{m} b_{i, 0,0}=\frac{1}{1-p} \times b_{0,0,0}
$$

Using the value of $b_{0,0,0}$ from Equation (13) and solving it further, Equation (14) can be written as the following.

$$
\tau=\frac{8}{(3 W+4)+3 p \times W \times \sum_{i=0}^{m-1}(2 p)^{i}}
$$

In general, transmission probability $\tau$ depends on the unknown value of $p$. Hence, to find the conditional collision probability $p$, probability $p$ is the probability that, in a given time slot, at least one of the other $n-1$ remaining STAs are transmitting, which can be written as follows.

$$
p=1-(1-\tau)^{n-1}
$$


Equations (15) and (16) represent a nonlinear system for two unknowns $\tau$ and $p$, which can be solved numerically for each other. It is easy to prove that this system has a unique solution:

$$
\tau(p)=1-(1-p)^{\frac{1}{n-1},}
$$

which is a continuously increasing function in the range of $p \in(0,1)$. Equations (15) and (17) show that the transmission probability depends on the network parameters such as contention window size, backoff stage limit, and the contending number of STAs. The size of network $n$ cannot be directly controlled, as none of the STAs in the network would know other contending STAs. Thus, the only method to find the optimal value for $\tau$ is to adaptively tune the values of $W, m$, and $n$ [15].

\subsection{Throughput Analysis}

We assume normalized throughput of a network as $S$ and characterized it as the amount of time during which the channel is utilized to effectively transmit data packets. To process the value of $S$, let us break down what occurs in a randomly selected transmission slot time. Subsequently, we expect that $P_{t r}$ is the likelihood that there is at least one device transmitting in the given slot time among an $n$ number of contending STAs, and it is given by the following.

$$
P_{t r}=1-(1-\tau)^{n}
$$

Probability $P_{s}$ for a successful transmission is given by the probability that exactly one STA transmits and the remaining STAs defer transmission, and it is given by the following.

$$
P_{s}=\frac{\left(n \times \tau \times(1-\tau)^{n-1}\right.}{1-(1-\tau)^{n}}
$$

According to the definition of the throughput, we compute throughput $S$ as the following ratio:

$$
S=\frac{E[\text { payload information transmitted in a given slot time }])}{[\text { Length of a slot time }]}
$$

Let $E[P]$ be the expected data packet size; thus, the average amount of payload information successfully transmitted in a slot time can be expressed as $P_{t r} \times P_{S} \times E[P]$ since an effective transmission happens in a slot time $P_{t r} \times P_{s}$. The normal length of the slot time is achieved with $1-P_{t r}$, and the empty slot time with probability $P_{t r} \times P_{S}$ contains a successful transmission. With probability $P_{t} r \times\left(1-P_{S}\right)$, it contains a collided packet transmission. Hence, throughput $S$ can be expressed as the following.

$$
S=\frac{P_{t r} \times P_{S} \times E[P]}{\left(1-P_{t r}\right) \times \sigma+P_{t r} \times P_{S} \times T_{S}+P_{t r} \times\left(1-P_{S}\right) \times T_{C}}
$$

Here, $T_{S}$ represents the time at which the wireless channel was detected to be occupied (that is, the slot time keeps going) on the account of a successful transmission, and $T_{C}$ represents the time at which the wireless channel was detected to be occupied by each STA at the time of collision. Here, $\sigma$ shows the duration of a vacant slot time. Obviously, values $E[P], T_{S}, T_{C}$, and $\sigma$ need to be expressed with a similar unit of estimation. The throughput calculation in (20) was acquired without the need to indicate the employed channel access algorithm. To explicitly process the throughput for a given DCF channel access mechanism, it is vital just to indicate the relating values of $T_{S}$ and $T_{C}$. In this manner, let us initially consider a WLAN network that is completely managed by means of the fundamental channel access mechanism of DCF (it is assumed to simplify our validation of the model). Let $H=P H Y_{\text {header }}+M A C_{\text {header }}$ be the data packet header for PHY and MAC layers 
and $\delta$ be the propagation delay. For basic access mechanism $T_{S}$ and $T_{c}$, we can obtain the following.

$$
\begin{gathered}
T_{S}=H+E[P]+S I F S+A C K+D I F S+2 \times \delta \\
T_{\mathcal{C}}=H+E[P]+D I F S+2 \times \delta
\end{gathered}
$$

\section{Model Validation}

In this section, we validate the proposed ReBOCA model with analytical results obtained on the basis of the specific MAC layer and PHY layer parameters as shown in Table 1. To evaluate the performance of ReBOCA, we compare simulation results with conventional BEB mechanism and one of the recently proposed related COSB algorithm [13]. The comparison protocol COSB was selected because it does not reset the value of $C W$ to its minimal value $C W_{\text {min }}$. COSB uses a channel observation-based scaled $C W$ instead of resetting to $\mathrm{CW}_{\min }$ after successful transmission. The COSB protocol increases or decreases the $C W$ value on the basis of channel collision probability. We validated the analytical model with $N=5,10,15,20,25,30,35,40,45,50$ number of STAs in a WLAN network, where each STA is within the coverage area of transmission range of each other to create a nonhidden terminal situation. The STAs always transmit, that is, they are in the saturated network situation.

Table 1. Evaluation parameters and their values.

\begin{tabular}{ll}
\hline Parameter & Value(s) \\
\hline Frequency & $2.4 \mathrm{GHz}$ \\
Bandwidth & $20 \mathrm{MHz}$ \\
Data payload & 1024 Bytes \\
ACK size & 16 Bytes \\
$C W_{\text {min }}$ & 32 \\
COSB $\omega$ & 32 \\
SIFS & $16 \mu \mathrm{s}$ \\
DIFS & $60 \mu \mathrm{s}$ \\
Propagation & $1 \mu \mathrm{s}$ \\
Data rate & $54 \mathrm{Mbps}$ \\
CW & 1024 \\
$\sigma$ & $9 \mu \mathrm{s}$ \\
Maximum backoff stages & 6 \\
\hline
\end{tabular}

Figure 4 shows the increase in channel collision due to the increase in the number of contending STAs. As shown in the figure, collision among the stations increases as congestion in the network increases, which is obvious due to the increase in transmission attempt. The conventional backoff algorithm BEB struggles to handle this situation and eventually results in a higher collision, especially for denser environments. The blind increase and decrease in a contention window in BEB algorithm raises the issue of collision for this protocol, especially resetting its $\mathrm{CW}$ to zero after successful transmission. At the same time, the COSB protocol works well to reduce collision situation in a network due to its channel-observation-based strategy. However, COSB depends on variance in the channelobservation-based collision probability due to the random channel access mechanism, which increases error variance due to the increase in recursive backoff stage visits. Our proposed sliding group window-based backoff mechanism, that is, ReBOCA, further decreases network collision due to the slower sliding of the contention size instead of resetting or changing it to a larger size. The proposed ReBOCA protocol approximately reduces $41.015 \%$ and $10.11 \%$ channel collision probability than compared to BEB and COSB, respectively. The decrease in channel collision probability in ReBOCA eventually results in higher probability to transmit a data packet, as shown in Figure 5. The lower transmission probability $(\tau)$ of ReBOCA than compared to that of COSB is mainly due to gradual slide-up (increase) in the $\mathrm{CW}$, whereas COSB directly changes the size of $\mathrm{CW}$ on the basis of channel collision probability. However, with the increase in the contending STA, 
the transmission probability for ReBOCA STAs starts increasing when compared to BEB. The decrease in collision probability $(p)$ and the increase in transmission probability $(\tau)$ enhance the ability to successfully transmit data. In addition, the increase in transmission probability may cause higher channel collision due to excessive data-packet transmissions. However, ReBOCA still maintains better performance due to the gradual distribution of contending devices within the contention parametric distribution.

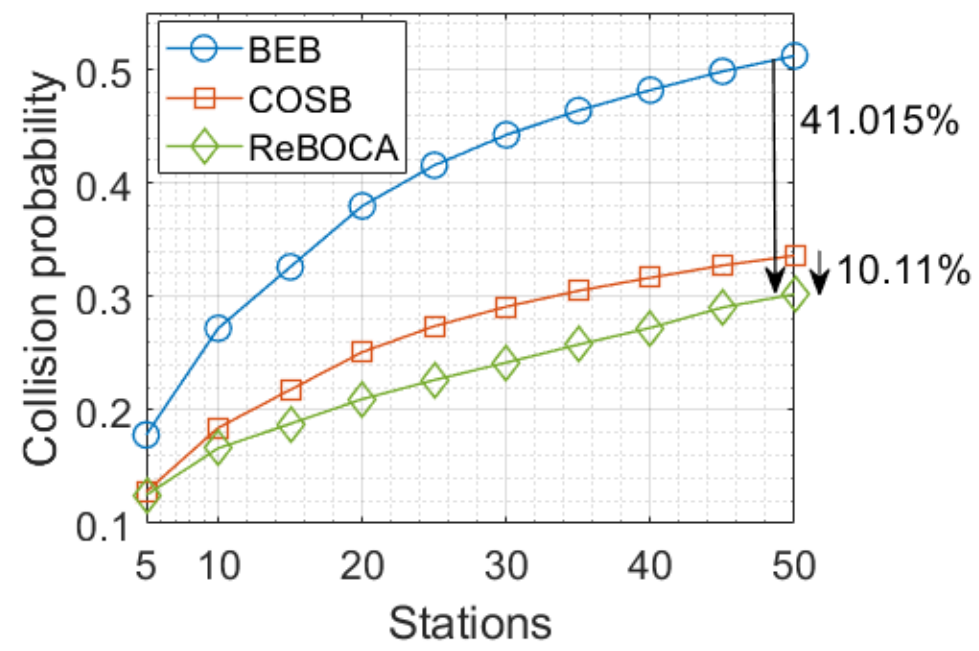

Figure 4. Comparison of collision probability $(p)$ among BEB, COSB, and ReBOCA due to change in network density.

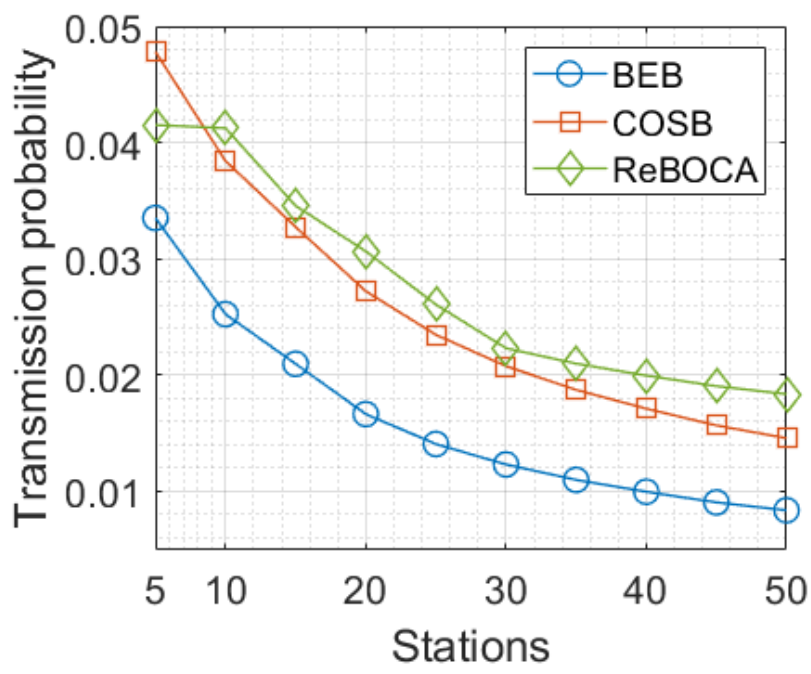

Figure 5. Comparison of transmission probability $(\tau)$ among BEB, COSB, and ReBOCA due to change in network density.

In Figure 6, we compare the resultant successful transmission probability $\left(P_{s}\right.$, as of Equation (19)) due to reduced collision and increased transmission probability. As depicted in Equation (19), a higher value of $\tau$ provides a higher probability of successful transmission. Furthermore, this increase in successful transmission eventually enhances the overall throughput of the WLAN network, as shown in Figure 7. In this figure, we compare the normalized throughput among BEB, COSB, and the proposed ReBOCA mechanism. A decrease in channel collision and increase in successful transmission eventually result in enhanced network throughput because each STA has much more time and opportunity to transmit as it finds collision-free slots more often. Our proposed ReBOCA mechanism enhances the normalized throughput of the dense WLAN network by $38.18 \%$ and $9.58 \%$ than compared to BEB and COSB, respectively. If we closely observe and compare the 
percentage of decreased channel collision (Figure 4) and the percentage of increased normalized throughput of the network (Figure 7), we can conclude that the throughput of the network is directly proportional to the collision within the network. Thus, decreasing collision increased throughput.

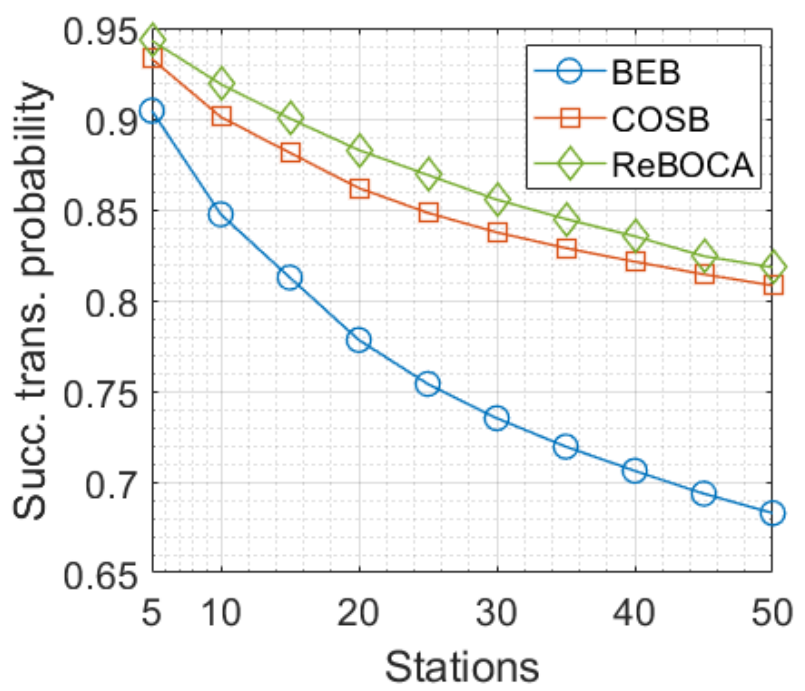

Figure 6. Comparison of probability of successful transmission $\left(P_{s}\right)$ among BEB, COSB, and ReBOCA due to change in network density.

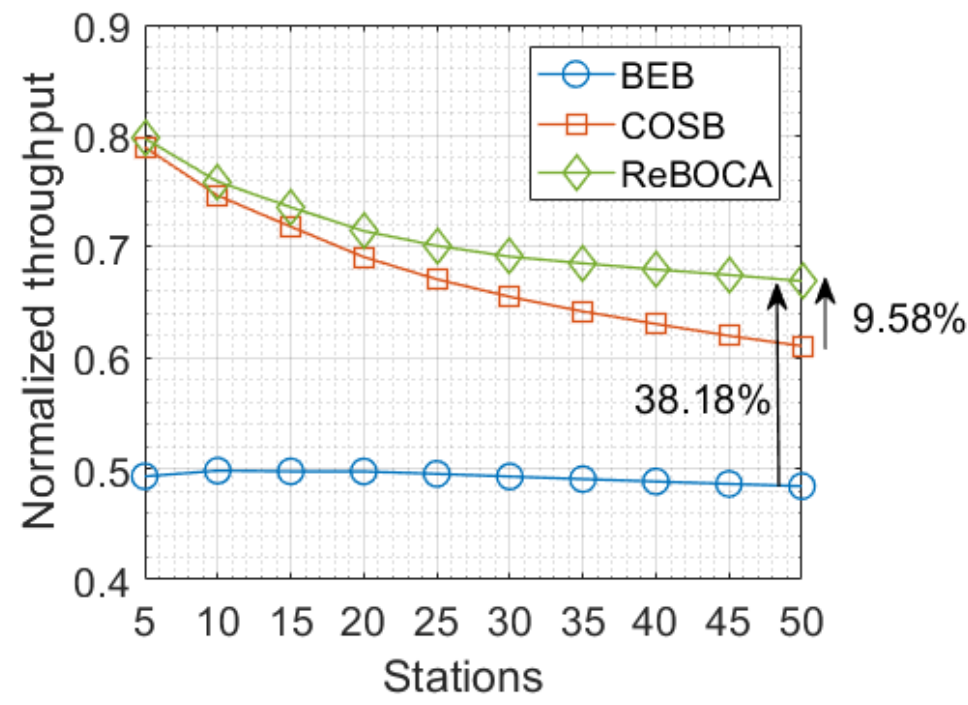

Figure 7. Comparison of normalized throughput among BEB, COSB, and ReBOCA due to change in network density.

\section{Computational Complexity}

The network-throughput improvement confirms the abilities of the proposed ReBOCA mechanism. However, this increased throughput results in a cost of computational complexity, which is obvious due to sliding group mechanism integration into the contention process where a STA might spend some of its time before accessing the channel resources. In Figure 8, we calculated the time complexity of ReBOCA with a standard BEB mechanism by running 1000 instances of the simulation. The figure shows that the computational time of the ReBOCA mechanism is slightly higher than that of the BEB mechanism, which is due to the requirement of the sGW procedure. The sliding group window mechanism in ReBOCA requires extra steps to optimize the performance of the backoff mechanism. 
These additional steps are the reason for the increase in the computational complexity of the ReBOCA mechanism.

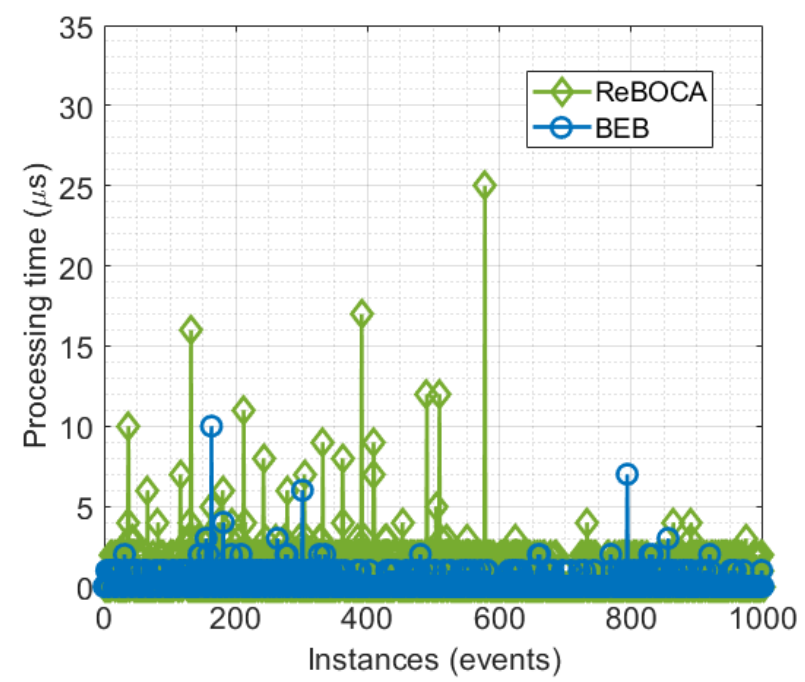

Figure 8. Comparison of computational complexity between our proposed ReBOCA mechanism and the conventional BEB mechanism.

\section{Conclusions and Future Work}

Recently, the IEEE 802.11ax WLAN was launched, promising high efficiency four times higher for denser environments. One of the bottlenecks for the WLAN remains as it faces the huge challenge of channel collision based on the CSMA/CA mechanism. CSMA/CA uses a BEB mechanism for contention resolution. In this paper, we proposed a rebacking-off for collision avoidance (ReBOCA) mechanism with a sliding group window, which converges the uniform distribution of the contention mechanism. The proposed variant of the conventional backoff mechanism allows for a fair sliding of the contending groups in the case of collisions. The key aspects of the ReBOCA mechanism include collision-point estimation, rebacking off for collision avoidance, and adaptive sliding of group windows to mitigate contention unfairness. An improvement in network throughput with lower channel collision probability was achieved by using the proposed mechanism, and efficiency increased as the number of contending STAs increased when compared to conventional CSMA/CA performance. Our proposed ReBOCA mechanism enhanced $38.18 \%$ network throughput when compared to that of a conventional BEB mechanism.

Author Contributions: Conceptualization, methodology, and validation, A.O.A. and R.A.; formal analysis, resources, data curation, Y.D.A.-O. and H.M.O.; writing-original draft preparation, A.O.A. and R.A.; writing-review and editing, Y.D.A.-O. and H.M.O.; supervision and project administration, T.K.; funding acquisition, A.O.A. All authors have read and agreed to the published version of the manuscript.

Funding: This research work was funded by Institutional Fund Projects under grant no. IFPHI-217611-2020.

Institutional Review Board Statement: Not applicable.

Informed Consent Statement: Not applicable.

Data Availability Statement: No new data were created or analyzed in this study. Data sharing is not applicable to this article.

Acknowledgments: This research work was funded by Institutional Fund Projects under grant no. IFPHI-217-611-2020. Therefore, the authors gratefully acknowledge technical and financial support from the Ministry of Education and King Abdulaziz University, Jeddah, Saudi Arabia.

Conflicts of Interest: The authors declare no conflict of interest. 


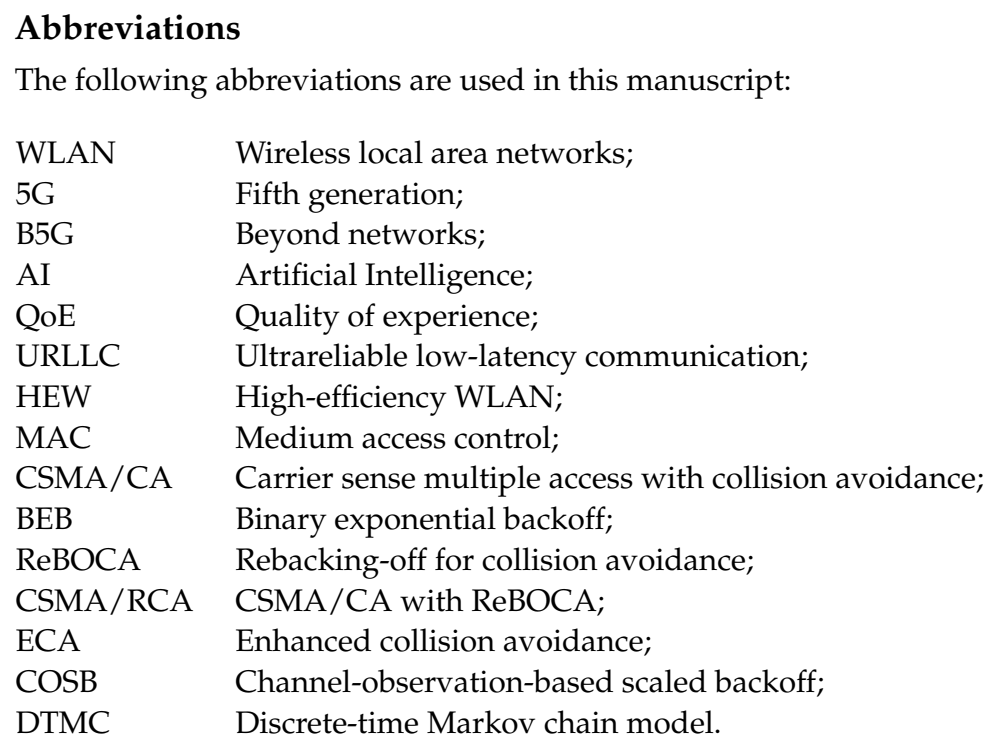

\section{References}

1. Ali, R.; Zikria, Y.B.; Bashir, A.K.; Garg, S.; Kim, H.S. URLLC for 5G and Beyond: Requirements, Enabling Incumbent Technologies and Network Intelligence. IEEE Access 2021, 9, 67064-67095. [CrossRef]

2. Bellalta, B. IEEE 802.11ax: High-efficiency WLANs. IEEE Wirel. Commun. 2016, 23, 38-46. [CrossRef]

3. Bioglio, V.; Condo, C.; Land, I. Design of Polar Codes in 5G New Radio. IEEE Commun. Surv. Tutor. 2020, 23, 29-40. [CrossRef]

4. Dai, L.; Fang, Y.; Yang, Z.; Chen, P.; Li, Y. Protograph LDPC-Coded BICM-ID with Irregular CSK Mapping in Visible Light Communication Systems. IEEE Trans. Veh. Technol. 2021. [CrossRef]

5. Xu, L.; Wang, H.; Gulliver, T.A. Outage Probability Performance Analysis and Prediction for Mobile IoV Networks Based on ICS-BP Neural Network. IEEE Internet Things J. 2021, 8, 3524-3533. [CrossRef]

6. Ali, R.; Shahin, N.; Kim, Y.T.; Kim, B.; Kim, S.W. Channel Observation-based Scaled Backoff Mechanism for High Efficiency WLANs. Electron. Lett. 2018, 54, 663-665. [CrossRef]

7. Ali, R.; Kim, S.W.; Kim, B.; Park, Y. Design of MAC Layer Resource Allocation Schemes for IEEE 802.11ax: Future Directions. IETE Tech. Rev. 2018, 35, 28-52. [CrossRef]

8. IEEE Standard for Information Technology. Part 11: Wireless LAN Medium Access Control (MAC) and Physical Layer (PHY) Specifications. ANSI/IEEE Std 802.11 2007. [CrossRef]

9. Min-seok, O. IEEE 802.11ax High Efficiency WLAN (HEW) Standardization Trend. OSIA Stand. Technol. Rev. 2014, 27, 16-23. Available online: https:/ / www.earticle.net/Article/A273769 (accessed on 29 August 2021).

10. Sanabria-Russo, L.; Barcelo, J.; Bellalta, B.; Gringoli, F. A High Efficiency MAC Protocol for WLANs: Providing Fairness in Dense Scenarios. IEEE/ACM Trans. Netw. 2017, 25, 492-505. [CrossRef]

11. Sanabria-Russo, L.; Faridi, A.; Bellalta, B.; Barcelo, J.; Oliver, M. Future evolution of CSMA protocols for the IEEE 802.11 standard. In Proceedings of the 2013 IEEE International Conference on Communications Workshops (ICC), Budapest, Hungary, 9-13 June 2013; pp. 1274-1279.

12. Ye, C.; Li, Y.; Reznik, A. Performance Analysis of Exponential Increase Exponential Decrease backoff Algorithm. In Proceedings of the 2010 IEEE Global Telecommunications Conference (GLOBECOM), Miami, FL, USA, 6-10 December 2010.

13. Ali, R.; Shahin, N.; Bajracharya, R.; Kim, B.-S.; Kim, S.W. A Self-Scrutinized Backoff Mechanism for IEEE 802.11ax in 5G Unlicensed Networks. Sustainability 2018, 10, 1201. [CrossRef]

14. Ali, R.; Zikria, Y.B.; Amin, F.; Kim, B.; Kim, S.W. I-DTMC: An Integrated-Discrete Time Markov Chain Model for Performance Analysis in Future WLANs. In Proceedings of the 2017 IEEE 42nd Conference on Local Computer Networks Workshops, Singapore, 9-12 October 2017.

15. Bianchi, G. Performance analysis of the IEEE 802.11 distributed coordination function. IEEE J. Sel. Areas Commun. 2000, 18, 535-547. [CrossRef] 